\title{
Performance Analysis of Transmit Antenna Selection with MRC in MIMO for Image Transmission in Multipath Fading Channels Using Simulink
}

\author{
Vaibhav S. Hendre*, **, M. Murugan***, Sneha Kamthe* \\ * Department of Electronics and Telecommunication Engineering, Trinity College of Engineering, University of Pune \\ ** Research Scholar of Sathyabama University, Chennai, Tamilnadu. India \\ *** Electronics and Communication Engineering Department at SRM's Valliammai Engineering College, Chennai \\ (e-mail:hendrevaibhav@gmail.com),(e-mail:dr.murugan.m@gmail.com),(e-mail: snehakamthe@gmail.com)
}

\begin{tabular}{l} 
Article Info \\
\hline Article history: \\
Received Oct 10, 2014 \\
Revised Nov 20, 2014 \\
Accepted Dec 13, 2014 \\
\hline Keyword: \\
Bit Error Rate (BER) \\
Maximal Ratio Combining \\
(MRC) \\
Multiple Input Multiple Output \\
(MIMO) \\
Orthogonal Space Time Block \\
Coding (OSTBC) \\
Transmit Antenna Selection \\
(TAS)
\end{tabular}

\begin{abstract}
Multiple antenna configurations can be used to increase the data throughput reducing the effects of multipath fading and interference when channel bandwidth is limited. Orthogonal Space Time Block Codes along with Transmit antenna selection can improve the performance of multiple input multiple output systems. In this paper, we present the Transmit Antenna Selection (TAS) technique based on the Maximal Ratio Combining (MRC) scheme with single antenna selection for image transmission. The performance analysis of the system was carried out under different fading channels i.e. Rayleigh and Rician channel for image input. We design end to end TAS/MRC system in Simulink with advancements in the channel designs and receive diversity techniques along with the feedback models. The Bit Error Rate (BER) analysis was performed for the combinations of number of transmit and receive antennas for TAS/MRC system for various fading environments.
\end{abstract}

Copyright (C) 2015 Institute of Advanced Engineering and Science. All rights reserved.

\section{Corresponding Author:}

Vaibhav S. Hendre,

Department of Electronics and Telecommunication,

Trinity College of Engineering and Research, Pune,

Savitribai Phule Pune University,

Maharashtra - 411048, India.

Email: hendrevaibhav@gmail.com

\section{INTRODUCTION}

The recent researches have shown that the large gains in capacity of communication over wireless systems along with the enhancement in system performance over conventional systems in multipath fading channels can be obtained by using Multiple Input Multiple Output (MIMO) [1]. Space Time Block Codes (STBCs) have its application in cellular communications and wireless local area networks. MIMO systems along with Space time block codes have become a most vibrant research area aimed at incorporating true multimedia services in conventional systems [2]. The parameters that are considered while using suitable coding method are reliability, spectral efficiency and performance gain. STBC is a transmit strategy used in MIMO technique exploiting transmit diversity and high reliability. Orthogonal STBCs are class of linear STBCs and they ensure full diversity order Lt $\mathrm{x} \mathrm{Lr}$ where $\mathrm{Lt}$ is number of transmit antennas and Lr is number of receive antennas [3]-[4]. Antenna Selection (AS) provides the full diversity and spectral efficiency gains with reduced hardware complexity at transmitter/receiver end. In AS a subset of the antennas is selected based on the channel knowledge at transmitter and/or receiver [5]. However the diversity order using antenna 
selection remains same as in case of multiple antennas. Transmit Antenna Selection (TAS) could be adopted in uplink and downlink of all future wireless communication systems [6]. TAS needs the Channel State information (CSI) knowledge at transmitter end. Therefore, it is mandatory to estimate the channel conditions at receiver end and need to feedback to transmitter. The transmitter will select the subset out of Lt antennas available at transmitter end [7]. This channel knowledge is in the form of index that represents the best set of antennas that are fed back from the receiver [7] [8]. The Channel prediction is useful in maintaining the transmit diversity and improving the BER and outage performance. The Maximal Ratio Combining (MRC) is a receiver diversity combining technique used at receiver end which helps in estimating the channel conditions for transmit antenna selection. MRC also helps to maximize the output signal to noise ratio (SNR) at the receiver [9] [10].

Shihao Yan et al [11] demonstrated the TAS scheme examining tradeoff between feedback overhead and secrecy performance with optimal power allocation. Investigations on the STBC for the MIMO systems [12] and subsequent TAS analysis of MIMO multi relay network [13] has been the current research areas. Based on this, the field of TAS/MRC along with STBC, a new system can be designed and extended on the basis of the previous models [14]. However all the work done has been on binary/uncoded data. The important feature of this paper is that the same work as in [9] is further extended for the image data with further advancements in the channel designs and the receive diversity technique designs along with the feedback models. The bit error rate (BER) analysis for various fading environments like Rayleigh and Rician is carried out and the system performance has been tested under different fading channels namely Rayleigh and Rician.

Further this paper investigates the analysis of combinations of number of transmit and receive antennas such as $2 \times 4,4 \times 1,2 \times 2,3 \times 2,4 \times 2$ and $4 \times 4$ MIMO systems with single TAS and without TAS under the effect of different fading channels. It involves the analysis of transmit antenna selection with maximal ratio combining for image transmission with the main focus on BER performance of the System.

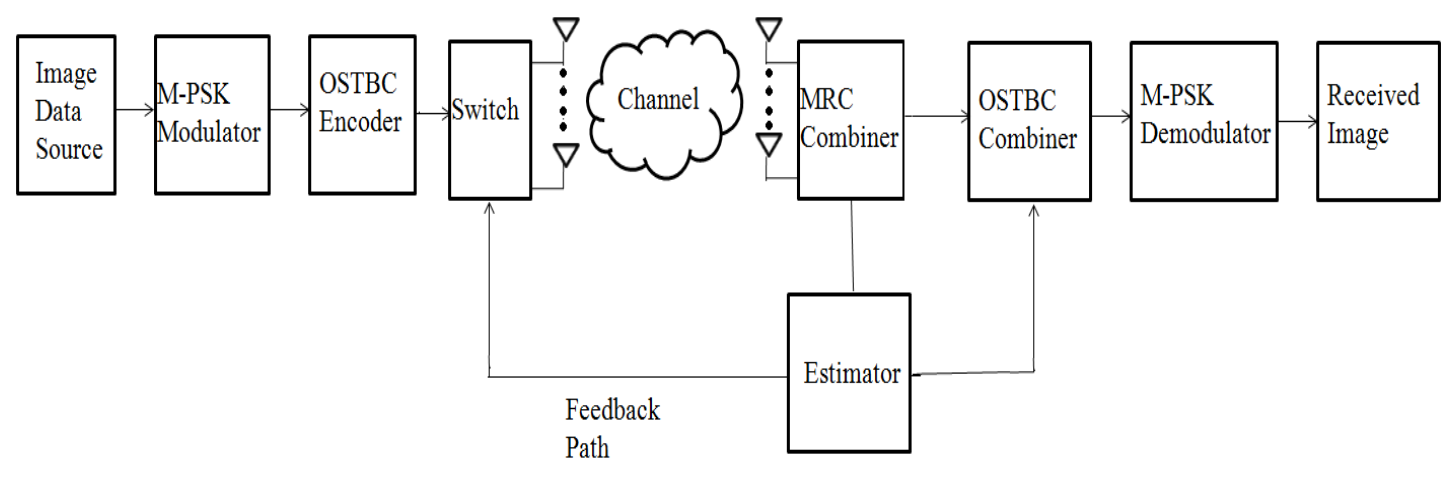

Figure 1. Basic System Block Diagram

The paper is organized as follows. Section II introduces the system model and channel model. Section III consists of error performance analysis of TAS/MRC scheme. Section IV includes block diagrams of various system models and sub models implemented in Simulink. Section V compares the BER analysis of MIMO schemes under different fading channel along with simulation results. Finally section VI presents the conclusion.

\section{SYSTEM AND CHANNEL MODEL}

Let a wireless link with a flat Rayleigh/Rician fading channel consisting $\mathrm{L}_{t}$ number of transmit antennas and $\mathrm{Lr}$ number of receive antennas. $\left(\mathrm{L}_{\mathrm{t}} ; \mathrm{L}_{\mathrm{r}}\right)$ system represents the system without antenna selection. The basic system model is shown in Figure 1. An image is transmitted over the channel. ' $\mathrm{H}$ ' is the channel matrix with dimensions $\mathrm{L}_{t} \times \mathrm{L}_{\mathrm{r}}$ and $\mathrm{h}_{\mathrm{i}, \mathrm{j}}$ are the channel fading coefficients where $1 \leq \mathrm{i} \leq \mathrm{L}_{\mathrm{t}}$ and $1 \leq \mathrm{j} \leq \mathrm{L}_{\mathrm{r}}$. These fading coefficients are the samples of Rayleigh/ Rician fading function. An $\left(\mathrm{L}_{\mathrm{r}} \mathrm{x} 1\right)$ vector is a column vector indicating channel between single transmit antenna and $\mathrm{Lr}$ receive antennas. It is assumed that the channel undergoes the quasi static fading i.e. the channel is constant for a block of transmission and this constant within block varies independently. This constant value is the combination of channel's constant gain 
and constant phase values. At any time instant $\mathrm{t}$, if the signal $\mathrm{x}$ is transmitted over the single selected antenna, the received signal vector can be expressed as

$$
y=h x+n
$$

where ' $y$ ' is received signal vector, ' $x$ ' is OSTBC encoded signal vector, ' $h$ ' is channel gain matrix and ' $n$ ' is noise vector.

A single selected transmit antenna, denoted by I, can be determined by equation as in [9].

$$
I=\underset{1 \leq i \leq L_{t}}{\operatorname{argmax}}\left\{C_{i}=\sum_{j=1}^{L_{r}}\left|h_{i, j}\right|^{2}\right\}
$$

which maximizes the total received signal power. We rearrange the random variables $\mathrm{Ci}, 1 \leq \mathrm{i} \leq \mathrm{Lt}$ in ascending order of magnitude where $C_{(1)} \leq C_{(2)} \leq \cdots \leq C_{\left(L_{t}\right)}$. According to (2), the transmit antenna corresponding to $C_{\left(L_{t}\right)}$ will be selected for transmission. The system model is based on two different fading channels Rayleigh and Rician fading channel model respectively.

\subsection{Rayleigh Channel Model}

The Rayleigh distribution has probability density function (PDF) given by [14]

$$
p_{R}(r)=\frac{2 r}{\Omega} e^{\frac{-r^{2}}{\Omega}}, r \geq 0
$$

where $r$ is the envelope of a sample of electric field and $\Omega$ is multiplication of in phase and quadrature component of electric field. The cumulative distribution function (CDF) for Rayleigh distribution is given by [14]

$$
F_{R}(r)=1-e^{\frac{-r^{2}}{\Omega}}
$$

where $\Omega=2 \sigma^{2}=E\left[r^{2}\right]$.

\subsection{Rician Channel Model}

The Rician distribution has probability density function (PDF) given by [14]

$$
p_{R}(r)=\frac{2 r}{\Omega} e^{\frac{-\left(A^{2}+r^{2}\right)}{\Omega}} I_{0}\left(\frac{2 A_{r}}{\Omega}\right), r \geq 0
$$

where $\mathrm{A}$ is the amplitude, $\mathrm{r}$ is the envelope of a sample of electric field and $\Omega$ is multiplication of in phase and quadrature component of electric field. The cumulative distribution function (CDF) for Rician distribution is given by [14]

$$
F_{R}(r)=1-Q_{1}\left(\frac{A}{\sqrt{\Omega / 2}}, \frac{r}{\sqrt{\Omega / 2}}\right)
$$

where $I_{0}($.$) is modified Bessel function of order 0$ and $Q_{1}($.$) is Marcum-Q function of order 1 . K_{r}=A^{2} / \Omega$ is Rician K-factor.

In this paper, the authors have considered single transmit antenna selection only. If at any time instant ' $t$ ' any one antenna of $\mathrm{L}_{t}$ transmit antennas is selected, then system is $\left(\mathrm{L}_{t}, 1 ; \mathrm{L}_{\mathrm{r}}\right)$ system. Here two cases for $\left(\mathrm{L}_{t}, 1 ; \mathrm{L}_{\mathrm{r}}\right)$ combination which are $(3,1 ; 2)$ and $(4,1 ; 2)$ respectively are considered. The $\left(\mathrm{L}_{t}, 1 ; \mathrm{L}_{\mathrm{r}}\right)$ system with TAS/MRC scheme for two channels, Rayleigh fading channel and Rician fading channel respectively is under consideration

\subsection{Error Performance Analysis TAS/MRC Scheme}

The Exact BER for TAS/MRC Scheme is presented here. For (Lt, 1; Lr) system with M-PSK modulation, the BER has been given by [9]

$$
\mathrm{P}=\int_{0}^{\infty} \mathrm{Q}\left(\sqrt{2 \gamma_{\mathrm{b}}}\right) \mathrm{p}_{\gamma_{\mathrm{b}}}\left(\gamma_{\mathrm{b}}\right) \mathrm{dr} \mathrm{r}_{\mathrm{b}}
$$


where $\gamma_{\mathrm{b}}=\gamma \cdot \mathrm{C}_{\left(\mathrm{L}_{\mathrm{t}}\right)}$ and $\mathrm{p}_{\gamma_{\mathrm{b}}}\left(\gamma_{\mathrm{b}}\right)=\frac{1}{\gamma} \mathrm{p}_{\left(\mathrm{L}_{\mathrm{t}}\right)}\left(\frac{\gamma_{\mathrm{b}}}{\gamma}\right)$.

Solving this expression by substituting the values, we get the exact BER expression for (Lt, 1 ; Lr) system with TAS/MRC system as

$$
\begin{gathered}
P=\frac{L_{t}}{L_{r}-1 !} \sum_{k=0}^{L_{t}-1}\left\{\frac{(-1)^{k}\left(\begin{array}{l}
\mathrm{L}_{t}-1 \\
k
\end{array}\right)}{[2(k+1)]^{L_{r}}}\right. \\
\left.\sum_{t=0}^{k\left(L_{r}-1\right)}\left[a_{t}\left(L_{r}, k\right)\left(L_{r}+t-1\right) !\left(1-\sqrt{\frac{\gamma}{\gamma+k+1}}\right)^{L_{r}+t} \sum_{j=0}^{L_{r}+t-1} 2^{-j}\left({ }^{L_{r}+t-1+j}\right)\left(1+\sqrt{\frac{\gamma}{\gamma+k+1}}\right)^{j}\right]\right\}
\end{gathered}
$$

The Average SNR Gain Comparison with Receiver MRC and STBCs is presented here. For the (Lt, 1 ; $\mathrm{Lr}$ ) TAS/MRC, the average SNR gain is the expected value of $\mathrm{C}_{\left(\mathrm{L}_{\mathrm{t}}\right)}$. We have the average SNR gain as [9]

$$
E\left\{C_{\left(L_{t}\right)}\right\}=\frac{L_{t}}{L_{r}-1 !} \sum_{k=0}^{L_{t}-1}(-1)^{k}\left({ }_{k}^{L_{t}-1}\right) \sum_{t=0}^{k\left(L_{r}-1\right)} \frac{b_{t}\left(L_{r}, k\right)\left(L_{r}+t\right) !}{(k+1)^{L_{r}+t+1}}
$$

For $\mathrm{Lr}=2$, the average SNR gain for the (Lt, 1; 2) TAS/MRC becomes [9]

$$
E\left\{C_{\left(L_{t}\right)}\right\}=L_{t} \sum_{k=0}^{L_{t}-1}(-1)^{k}\left(\begin{array}{r}
L_{t}-1 \\
k
\end{array}\right) \times \sum_{t=0}^{k} \frac{\left(\begin{array}{l}
k \\
t
\end{array}\right)(t+2) !}{(k+1)^{t+3}}
$$

For an (Lt, Lr) STBC, the average SNR gain at the combiner output is [9]

$$
E\left\{\frac{1}{L_{t}} \sum_{i=1}^{L_{t}} \sum_{j=1}^{L_{r}}\left|h_{i, j}\right|^{2}\right\}=L_{r}
$$

\section{THE PROPOSED METHOD}

\subsection{Basic System Block Diagram}

Figure 2 shows the basic system block diagram. It consists of seven sections: Image conversion, Modulator, Encoder, Channel, Decoder, Demodulator and Image reconstruction. The input to the system is an image from which R, G, B contents are extracted and are converted into integer values. A modulation scheme applied is M-ary PSK in order to have better spectral efficiency and noise and interference immunity. In this case, ' $M$ ' is taken to be 8 so that the image is mapped into $28=256$ symbols. Orthogonal Space Time Block Code (OSTBC) is used for encoding the symbols into the streams depending on the number of transmitting antennas. The $4 \times 4$ Rayleigh/ Rician fading channel models the streams along with the Adaptive White Gaussian Noise (AWGN).

The OSTBC combiner combines the signals from all the receive antennas to extract the information of symbols that were mapped using OSTBC encoder with the help of channel estimates. Demodulation followed by image reconstruction gives the final output for which BER analysis is performed. 


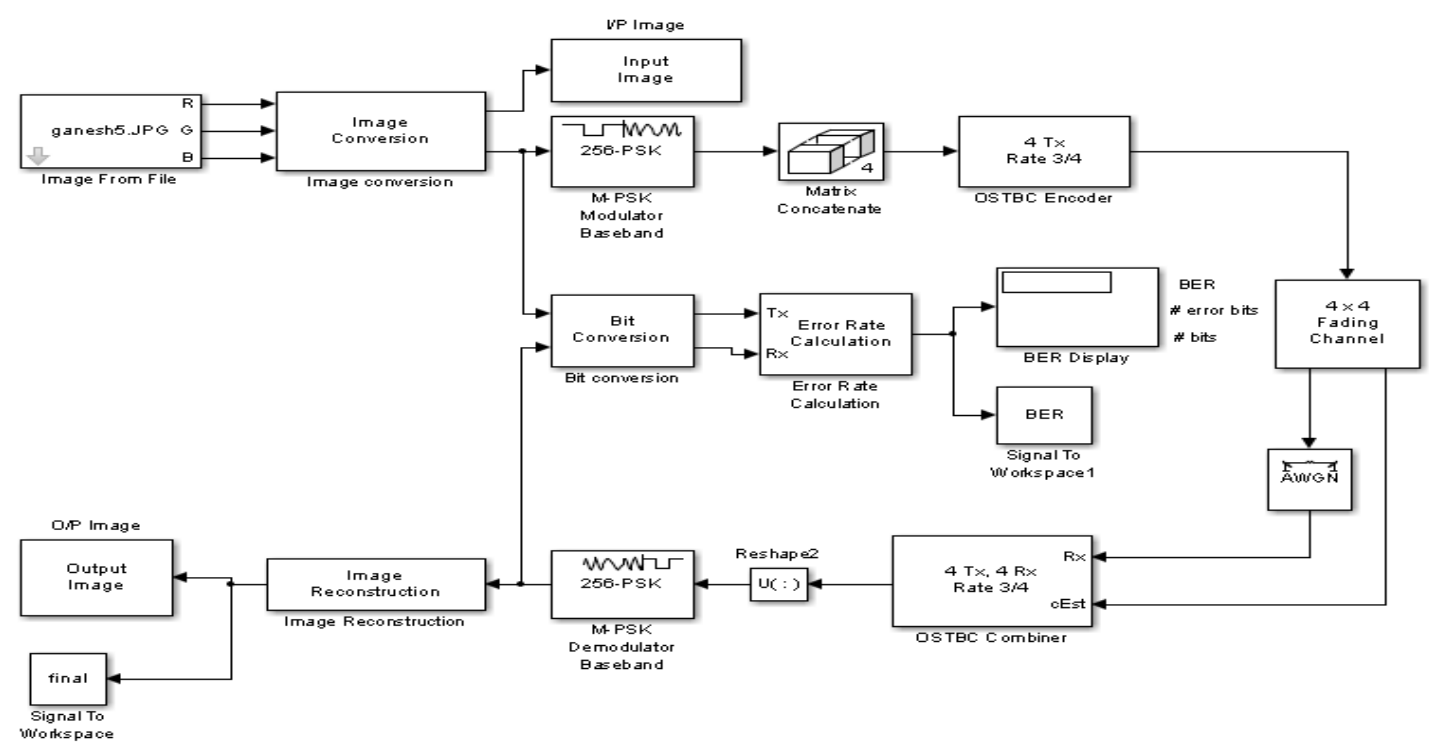

Figure 2. System Block Diagram in Simulink

\subsection{Transmit Antenna Selection}

A transmit antenna selection has been done based on the MRC value as shown in Figure 3. A switch case block from Matlab Simulink is used to perform the switching operation which uses MRC value to select a case condition that determines which subsystem will be executed. Each output port of switch case block is connected to switch case action subsystems. The case value that corresponds to the MRC value determines the subsystem that will be executed. The single selected transmit antenna, given by I, is determined by equation 2 .

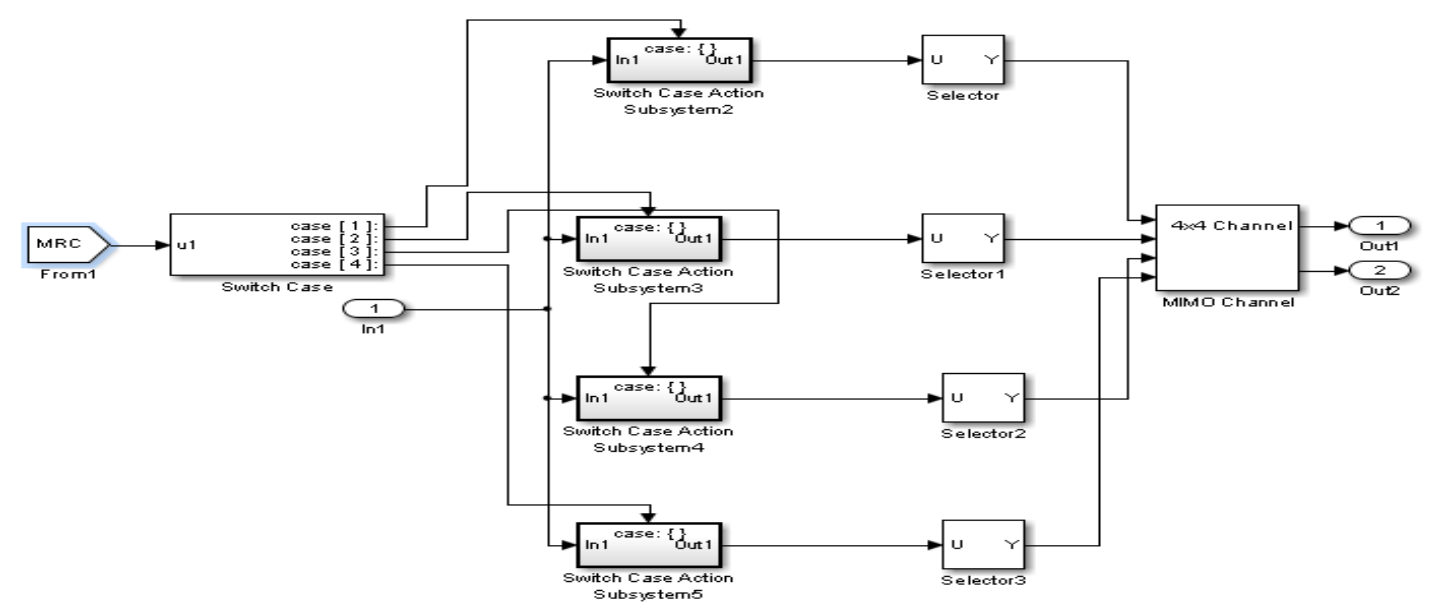

Figure 3. Transmit Antenna Selection based on Maximal Ratio Combining

\subsection{X4 MIMO Channel}

A Rayleigh fading channel shown in Figure 4 models the paths associated with signals transmitted from each of the transmitting antennas by multiplying the input signal by samples of Rayleigh distributed complex random process. The minimum number of paths existing between transmitter and receiver are dependent upon the number of receiving antennas. Each path has different Doppler shift, time delay vector, path gain vector. Path gains are required to calculate the MRC value of the path. The Authors have tried to create the multipath fading environment by designing the channel model in Simulink Environment as shown 
in Figure 4. The channel path for every input is considered to be the path for actual data transmission and the path for estimating the signal. The same model is repeated for other channels as shown in Figure 4.

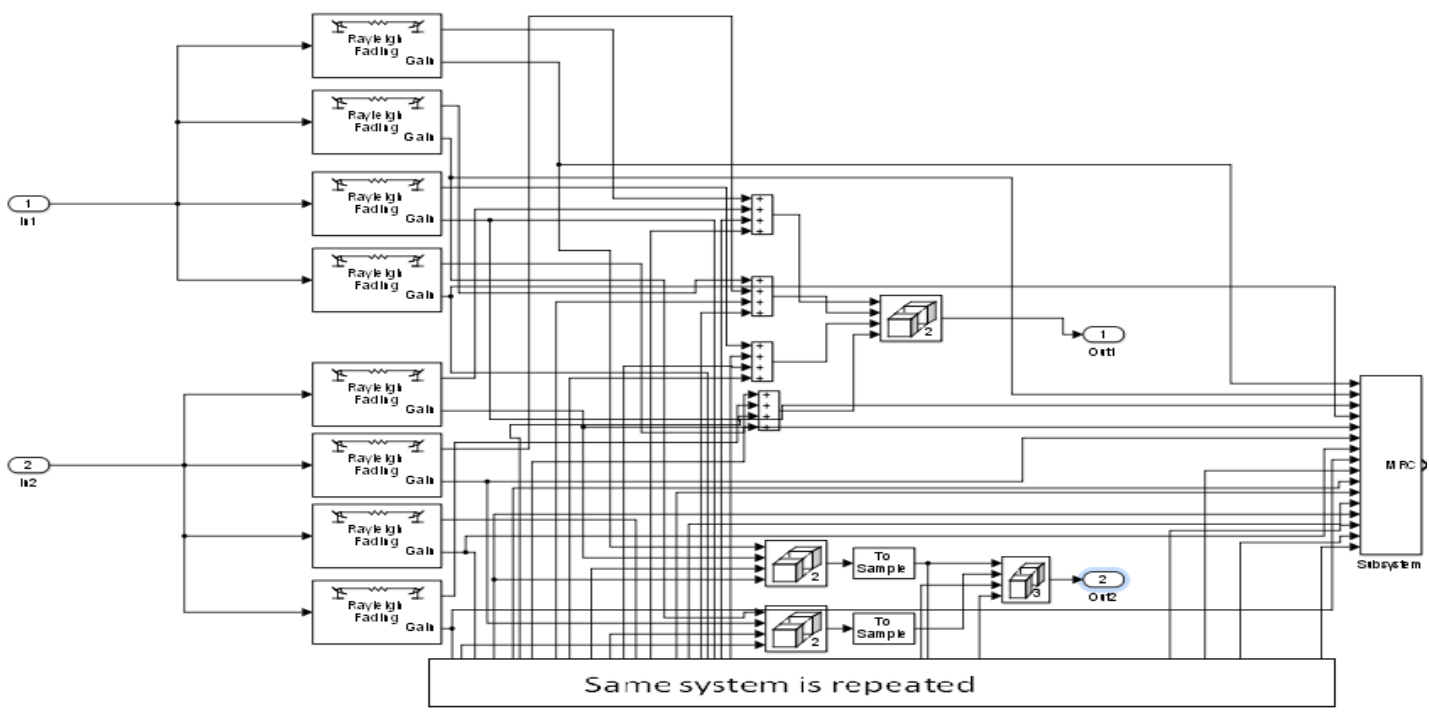

Figure 4. 4X4 MIMO Channel

\subsection{Maximal Ratio Combining}

Maximal Ratio Combining has been implemented by computing the value of $\mathrm{Ci}$ in equation (2). Matrix square block is used to compute the value of $\left|\mathrm{h}_{\mathrm{i}, \mathrm{j}}\right|^{2}$ and matrix sum block is used to add all the squared channel gains to generate $\mathrm{Ci}$ associated with that path. The index of the path with maximum $\mathrm{Ci}$ is feedback to the transmitter to select the appropriate transmit antenna with same index. Figure 5 shows the MRC technique for two receive antennas as implied in Simulink environment. Same structure can be extended further for more number of receive antennas. The model shown is for single input, same is repeated for other inputs.

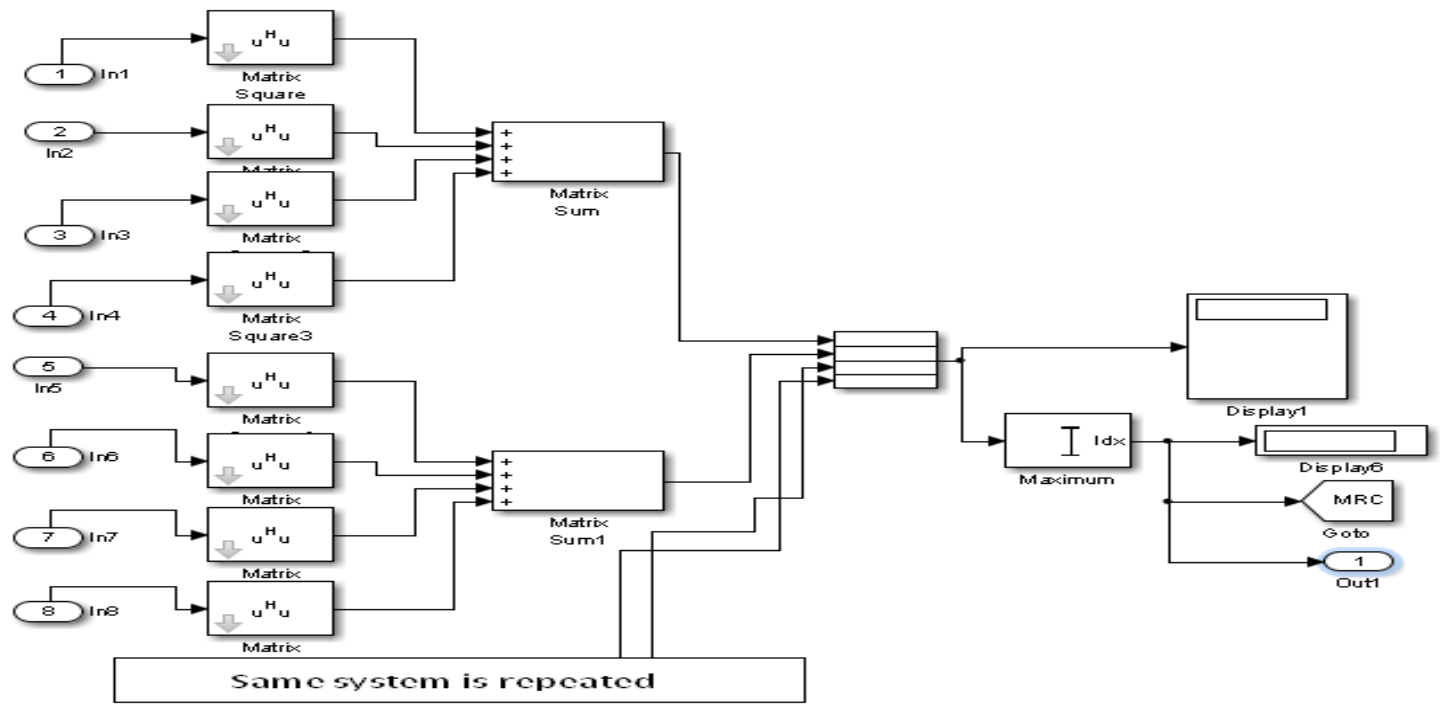

Figure 5. Maximal Ratio Combining 


\section{RESULTS AND ANALYSIS}

The performance of system has been analyzed for Bit Error Rate (BER) for various configurations such as $2 \times 2,3 \times 2$, and $4 \times 2$ along with TAS and without TAS for different fading channels.

\subsection{Rayleigh Channel}

Figure 6 shows the BER plot for 2x4, 4x1 MIMO without TAS and 4x4 MIMO with single TAS for Rayleigh fading channel. It can be seen that the system performance improves with transmit antenna selection for image input.

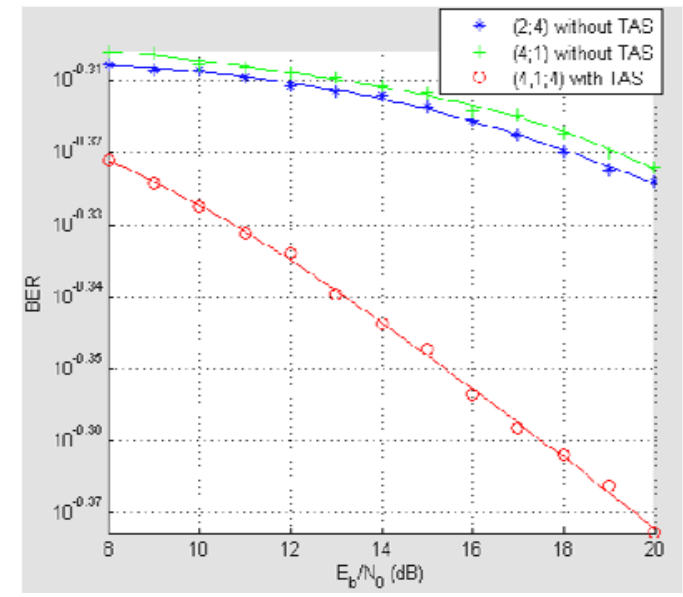

Figure 6. BER plot for $2 \times 4,4 \times 1$ MIMO configurations without TAS and $4 \times 4$ with single antenna selection

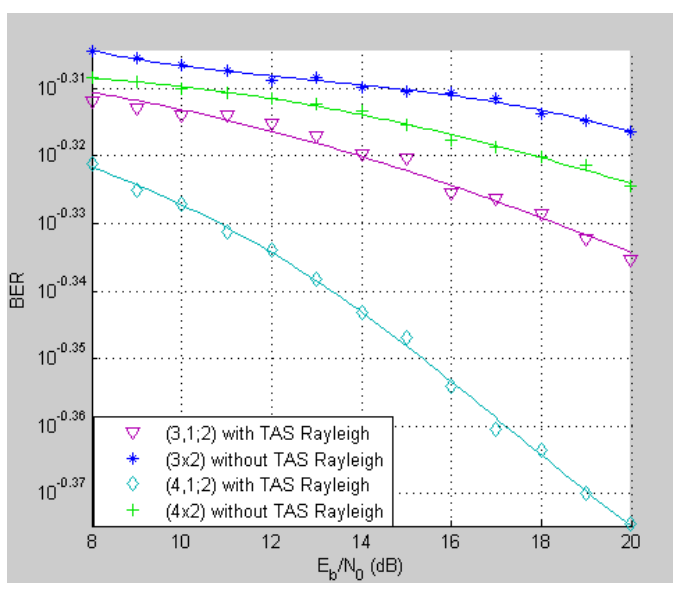

Figure 7. BER plots for $3 \times 2$ and $4 \times 2$ MIMO configurations with and without transmit antenna selection for Rayleigh fading channel

It has also been observed that BER decreases significantly with the change in the MIMO configurations. Figure 7 gives the comparison of MIMO with and without TAS for $3 \times 2$ and $4 \times 2$ MIMO configurations. It is observed from the graph that the significant change in BER for single TAS compared to conventional MIMO without TAS. It is also observed that as we go on increasing the number of transmit antennas the BER performance improves further. It is observed from Figure 8 that single antenna selection gives improved performance if we increase number of antennas at transmitter. Here number of receiving antennas are kept constant and number of transmit antennas are increased one by one, $(4,1 ; 2)$ performance is better than $(3,1 ; 2)$ and $(2,1 ; 2)$.

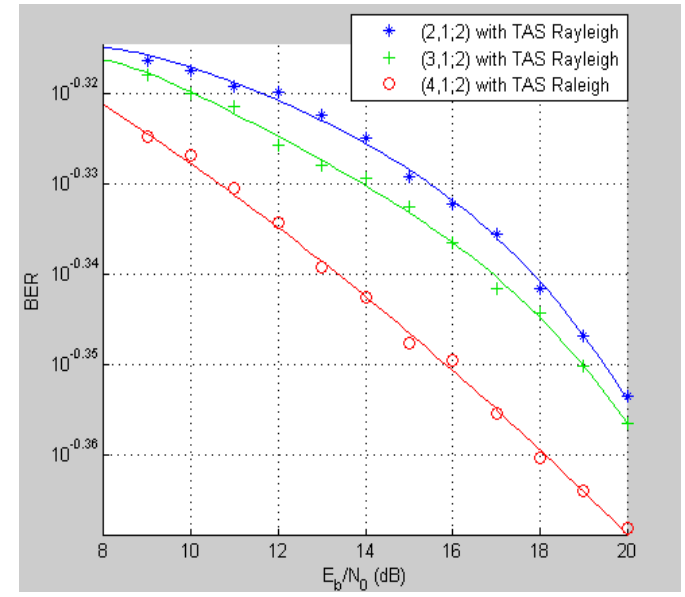

Figure 8 . BER plots for $2 \times 2,3 \times 2$ and $4 \times 2$ MIMO configurations with single transmit antenna selection for Rayleigh fading channel

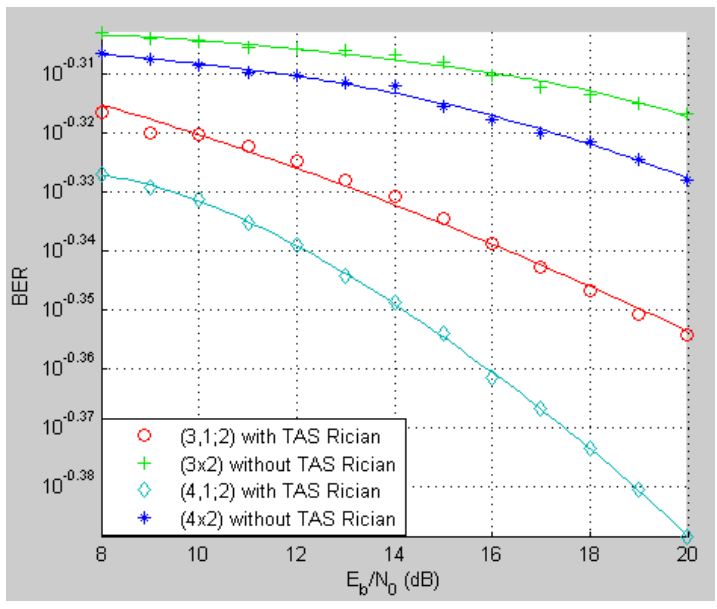

Figure 9. BER plots for $3 \times 2$ and $4 \times 2$ MIMO configurations with and without transmit antenna selection for Rician fading channel 


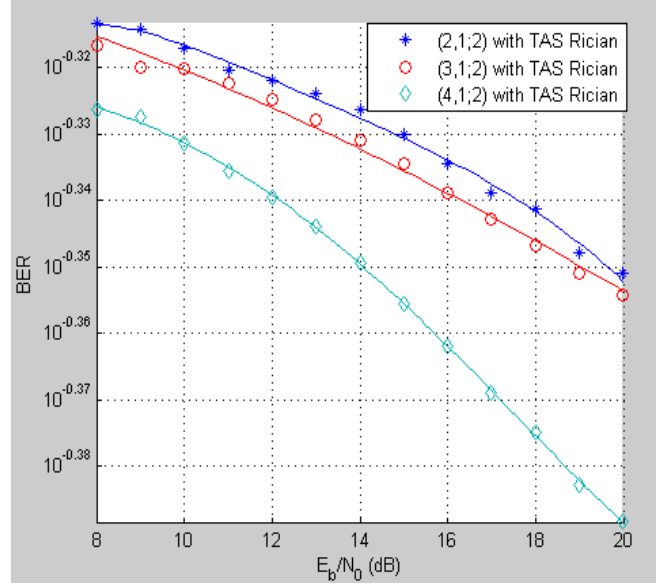

Figure 10. BER plots for $2 \times 2,3 \times 2$ and $4 \times 2$ MIMO configurations with single transmit antenna selection for Rician fading channel

\subsection{Rician Channel}

The comparison between $3 \times 2$ and $4 \times 2$ MIMO configurations with and without TAS is repeated for the Rician channel and as depicted in Figure 9. In the same manner, BER has been analyzed for different number of transmit antennas when number of receiving antennas are kept constant as shown in Figure 10. The results in case of Rician fading channel are better as compared to Rayleigh fading channel.

Table I shows the input and output images for each of the $2 \times 2,3 \times 2$ and $4 \times 2$ MIMO configuration and corresponding BER values without TAS and with TAS.SNR is kept constant to $10 \mathrm{~dB}$.

Table 1. Output Images for with and without TAS along with BER Values for SNR=10dB

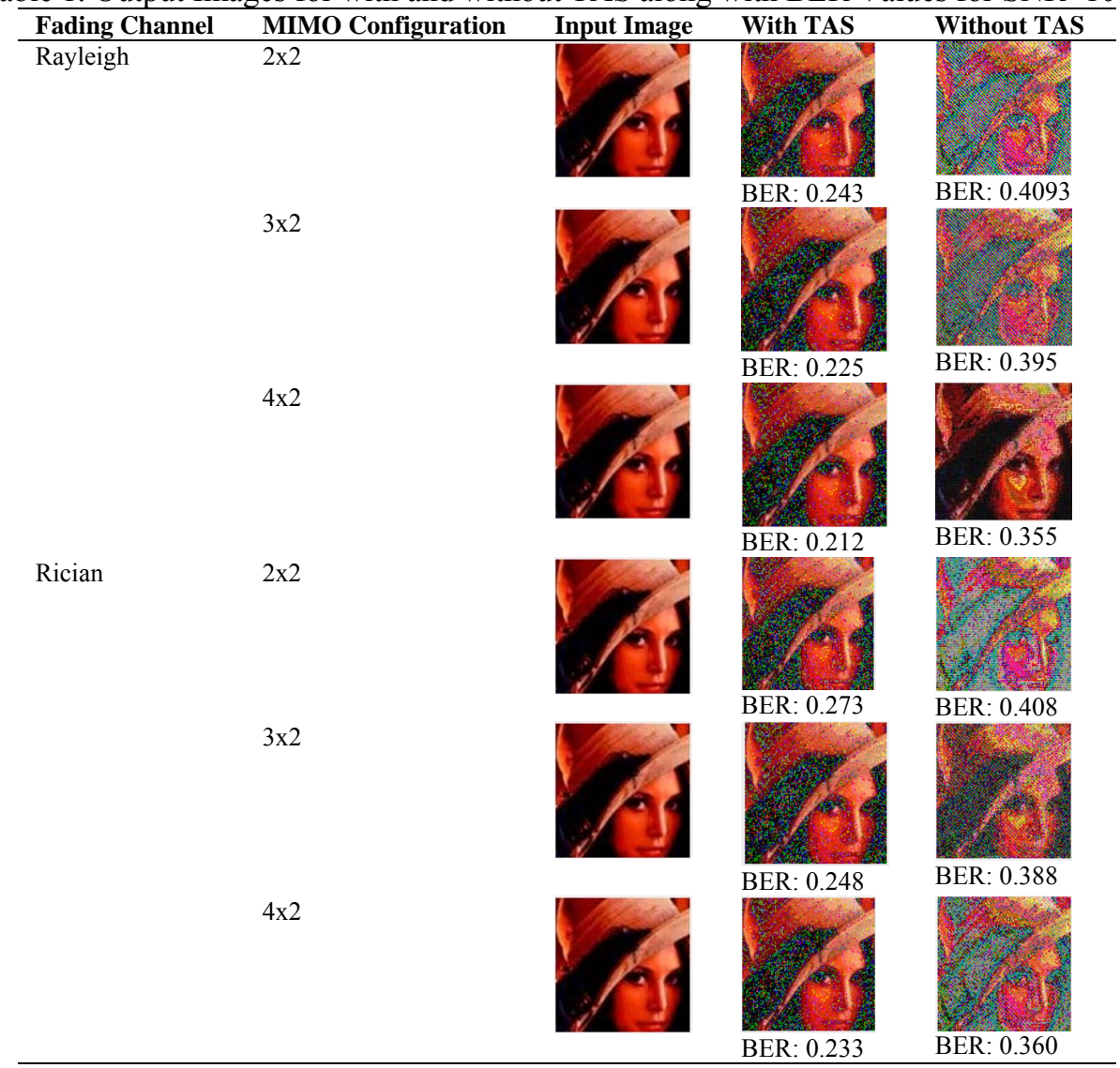




\section{CONCLUSION}

In this paper, performance analysis of transmit antenna selection with maximal ratio combining at receiver in MIMO Simulink environment with advanced channel models and feedback system. The authors have presented the bit error rate analysis for Rayleigh and Rician fading channel. It has been observed that the single transmit antenna selection gives improved performance compared to conventional MIMO systems for image transmission. It was also observed that system performance further improves if we increase number of transmit antennas. This work can be further extended for selecting more than one antenna in TAS with MRC. It can further possible to test the performance of system for advanced channel models defined in standards for wireless communication systems.

\section{REFERENCES}

[1] Muhammad Sana Ullah,Mohammed Jashim Uddin,"Performance Analysis of Wireless MIMO System by Using Alamouti's Scheme and Maximum Ratio Combining Technique", International Journal of Advanced Engineering Sciences and Technologies(IJAEST), Vol. 8, no. 1,pp.19-24, 2011.

[2] Santumon.S.D, B.R. Sujatha, "Space-Time Block Coding (STBC) for Wireless Networks", International Journal of Distributed and Parallel Systems (IJDPS),vol.3, no.4, July 2012.

[3] V.S. Hendre, M. Murugan, "A Performance of Precoded Orthogonal Space Time Block Code for Mobile Wi-MAX System", IEEE International Conference on Communications and Signal Processing (ICCSP-2013), IEEE Xplore ISBN: 978-1-4673-4865-2, p.no.5-8, 2013.

[4] Khoa T. Phan and Chintha Tellambura, "Capacity Analysis for Transmit Antenna Selection Using Orthogonal Space-Time Block Codes", IEEE Communications Letters, vol. 11, no. 5, May 2007.

[5] V.S. Hendre, M. Murugan, "A Comprehensive Literature Survey on Antenna Selection in MIMO for Future Generation Wireless Systems",CiiT International Journal of wireless Communication, WC 082012004, vol. 4, No. 13, August 2012.

[6] V.S. Hendre, M. Murugan, "Optimal Transmit antenna Selection for Closed Loop Spatial Multiplexed Precoded MIMO for LTE Systems", IETE National Journal of Innovation and Research, vol. 1, no. 2, pp. 22-25, January 2014.

[7] Shiva Prakash and Ian McLoughlin, "Effects of Channel Prediction for Transmit Antenna Selection with Maximal ratio Combining in Rayleigh Fading" IEEE Transactions on Vehicular Technology, vol. 60, no. 6, July 2011.

[8] Qinfang Sun, et al., "Estimation of Continuous Flat Fading MIMO Channels", IEEE Transactions on Wireless Communications, vol. 1, no. 4, October 2002.

[9] Zhuo Chen,Jinhong Yuan and Branka Vucetic, “Analysis of Transmit Antenna Selection/Maximal-Ratio Combining in Rayleigh Fading Channels", IEEE Transactions on Vehicular Technology, vol. 54, no. 4, July 2005.

[10] T.R. Ramya and Srikrishna Bhashyam, India. "Using Delayed Feedback for Antenna Selection in MIMO Systems", IEEE Transactions on Wireless Communications, vol. 8, no. 12, December 2009.

[11] Shihao Yan, et al., "Transmit Antenna Selection with Alamouti Coding and Power Allocation in MIMO Wiretap Channels", IEEE Transactions on Wireless Communications, vol. 13, no. 3, March 2014.

[12] Bilanja Bedic, "Space Time Block Coding for Multiple Antenna Systems", Ph.D. dissertation, Vienna Univ. of Technology, Austria, 2005.

[13] Phee Lep Yeoh, et al., "Unified Analysis of Transmit Antenna Selection in MIMO Multirelay Networks", IEEE Transactions on Vehicular Technology, vol. 62, no. 2, February 2013.

[14] Cyril-Daniel Iskander "A MATLAB -based Object-Oriented Approach to Multipath Fading Channel Simulation", http://www.mathworks.cn /matlabcentral/fx_files/18869/1/ChannelModelingWhitePaper.pd,,2009.

\section{BIOGRAPHIES OF AUTHORS}

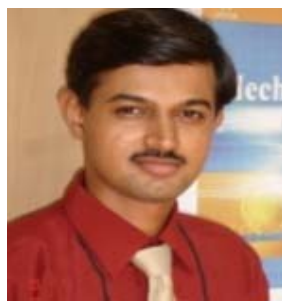

Vaibhav S Hendre received his B.E (Electronics and Telecommunication Engineering) from University of Pune in 1999 and M. Tech. from Dr. Babasaheb Ambedkar Technological University, Lonere in 2006. He is a registered Ph.D Research Scholar of Sathyabama University, Chennai-600119, Tamil Nadu, India. He is currently serving as Associate Professor and Head of Department of Electronics \& Telecommunication Engineering, Trinity College of Engineering \& Research, Pune-411048, and Maharashtra, India. Having 13 years of academic experience, his fields of interest are Error Control coding, Wireless Digital Communications and Signal Proceesing for MIMO systems. He is a member of IEEE Communication Society and Life Member of ISTE. 

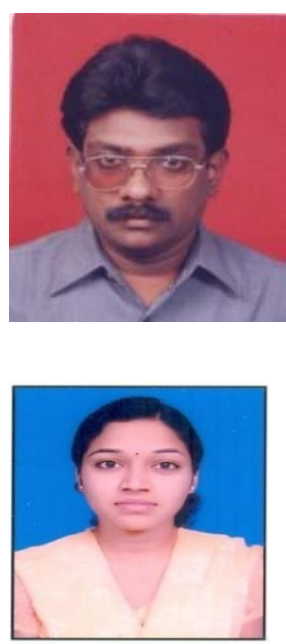

Dr. M. Murugan graduated in E\&CE from the University of Madras in 1989. Received his Masters in the E \& TC (Spl: Microwave) in 2001 and Ph.D in 2010 from the University of Pune. Presently he is the Professor, Head and Vice Principal of SRM's Valliammai Engineering College, Kattankulathur - 603203, T.Nadu, India. Having 25 years of experience in teaching, his fields of interests are Antennas, Microwave, Optical \& Satellite Communication and EMI\&C. $\mathrm{He}$ is a Fellow of IETE and Life Member of ISTE, IEI, ISOI, SEMCEI and Semiconductor Society (I). To his credit are over forty papers published in International / National repute Conferences/Journals.

Sneha S. Kamthe received her B.E. (Electronics \& Telecommunication Engineering) from University of Pune in 2012.She is pursuing her Masters of Engineering in Digital Systems from University of Pune. She is currently serving as lecturer in Electronics \& Telecommunication Department of Trinity College of Engineering \& Research, Pune-411048, Maharashtra, India. 